\title{
CARACTERIZAÇÃo DE CANAIS DE ACESSO EXTERNOS A ÁREAS PORTUÁRIAS BRASILEIRAS SEGUNDO AS RECOMENDAÇÕES DA PIANC - ANÁLISE DE LARGURAS
}

\author{
Paolo Alfredini e Reginaldo Galhardo Martins \\ Laboratório de Hidráulica da Escola Politécnica da Universidade de São Paulo \\ Av. Prof. Lúcio Martins Rodrigues, 120 - Cidade Universitária - CEP 05508-900 - São Paulo, SP \\ alfredin@usp.br
}

\section{RESUMO}

A sistematização do projeto de canais de acesso a áreas portuárias tem como escopo apresentar as recomendações e informações, que permitem formular o projeto conceitual de um canal para um navio-tipo, ou conjunto de navios, bem como analisar a adequação de um canal existente em função de modificações nas premissas de projeto, sempre tendo em vista a segurança da navegação. O projeto de um canal de acesso envolve fundamentalmente a definição das dimensões horizontais de alinhamento e largura e vertical de profundidade. A evolução da navegação comercial e de lazer exige o dimensionamento adequado de canais de acesso que evitem atrasos, congestionamentos, incidentes e acidentes, com sérias conseqüências econômicas. O projeto conceitual, ou básico, formulado em bases racionais que permitam a definição da largura do Canal de Acesso, constitui o primeiro estágio do projeto estabelecido a partir da base de dados ambientais disponivel, navio de projeto e outras condicionantes e previsões. Este artigo apresenta a caracterização de trinta canais de acesso externos dos principais portos comerciais brasileiros segundo as recomendações da PIANC.

\section{INTRODUÇÃO}

A sistematização do projeto conceitual de canais de acesso a áreas portuárias tem como objetivos apresentar as recomendações que permitam formular o projeto conceitual de um canal para um ou conjunto de navios-tipo, bem como analisar a adequação de um canal existente em função de modificações nas premissas do projeto, sempre tendo em vista a segurança da navegação. $O$ projeto de um canal de acesso envolve fundamentalmente a definição das dimensões horizontais de alinhamento e largura e verticais de profundidade. A evolução da navegação comercial e de lazer exige o dimensionamento adequado de canais de acesso que evitem atrasos, congestionamentos, incidentes e acidentes, com sérias conseqüências econômicas.

O projeto conceitual, ou básico, formulado em bases racionais que permitam a definição das dimensões horizontais e vertical do Canal de Acesso, constitui o estágio do projeto estabelecido a partir da base de dados ambientais disponível, navio de projeto e outras condicionantes e previsões. As recomendações da PIANC (1997), têm repercussões importantes no desenvolvimento de áreas portuárias por permitir estabelecer especificações que orientem de forma segura as dimensões da via navegável de acesso, bem como subsidiar a definição de critérios operacionais, tais como condições de navegação com mão dupla e situações que exijam dragagem.

\section{OBJETIVOS}

Com este trabalho objetivou-se a análise das larguras de canais externos, isto é expostos à agitação, dos principais portos da costa brasileira com intuito de se apresentar um panorama da realidade brasileira em relação aos critérios da PIANC (1997). A análise referida tem como princípio a comparação entre situações de pior caso e melhor caso, sendo elas determinadas a partir de uma probabilidade de ocorrência significativa, isto é, são considerados os casos piores e melhores que efetivamente apresentem um período de retorno baixo. O critério de classificação é baseado nas recomendações da PIANC (1997), que tratou dos elementos de canais de acesso, tanto internos como externos, através de considerações climáticas, físicas e da frota que freqüenta o porto.

Com esta análise apresenta-se uma avaliação de aplicabilidade das citadas recomendações aos portos brasileiros, visando contribuir na formulação de projetos básicos de canais de acesso com indicações preliminares úteis para estudos de viabilidade, bem como importante ferramenta para tomada de decisões operacionais referentes ao controle do tráfego marítimo. 


\section{DESCRIÇÃO DAS RECOMENDAÇÕES PIANC (1997)}

A determinação de larguras, segundo as recomendações PIANC (1997) é proporcional a um coeficiente multiplicativo da boca $(B)$ do navio-tipo, seguindo uma metodologia de discretização dos efeitos que apresentam influência significativa com relação à segurança das embarcação em um canal de acesso.

Os efeitos são:

- Manobrabilidade;

- Velocidade do Navio;

- Ventos Transversais Prevalecentes;

- Correntes Transversais Prevalecentes;

- Correntes Longitudinais Prevalecentes;

- Altura Significativa de Hs e Comprimento de Onda $(\lambda)$;

- Auxílios à Navegação;

- Superfície de Fundo;

- Profundidade de Via Navegável;

- Nível de Periculosidade da Carga;

- Intensidade de Tráfego;

- Largura Adicional devido à folga com a margem;

- Largura de Passagem para Canais de Mão Dupla.

A Figura 1 mostra de forma esquemática os efeitos acima.

\section{APRESENTAÇÃO DOS PORTOS ESTUDADOS}

A seguir são listados os portos estudados e as características do(s) respectivo(s) canal(is) de acesso e subseqüentemente na Figura 2 a sua distribuição geográfica:

- Belém - PA: o canal de acesso desenvolvese por cerca de $4 \mathrm{~km}$, com largura variando entre $90 \mathrm{~m}$ e $180 \mathrm{~m}$ e profundidade mínima de $6 \mathrm{~m}$.

- P. da Madeira/Itaqui - MA: o canal de acesso externo tem comprimento da ordem de $98000 \mathrm{~m}$, largura e profundidade mínimas de 500 e $24 \mathrm{~m}$.

- Mucuripe (Fortaleza) - CE: o canal de acesso, com extensão de $1,5 \mathrm{~km}$, possui largura variável entre $80 \mathrm{~m}$ e $100 \mathrm{~m}$ e profundidade de $10 \mathrm{~m}$.

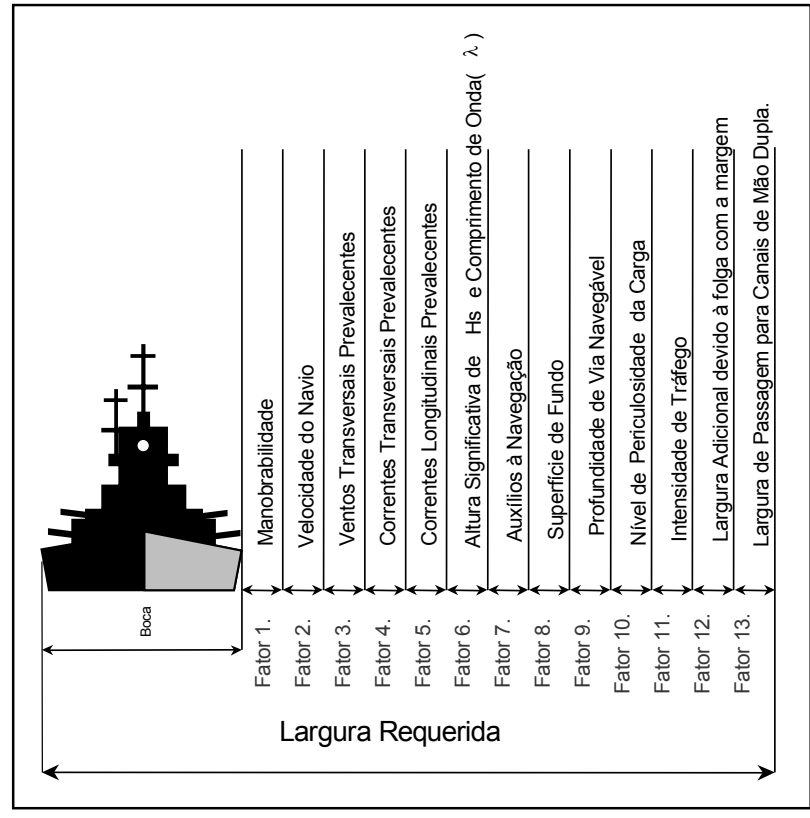

Figura 1. Discretização das parcelas constituintes do cálculo da largura requerida.

- Areia Branca - RN: localizado em mar aberto, o porto-ilha não possui barra definida. o canal de acesso tem comprimento aproximado de $15 \mathrm{~km}$, largura variável entre $400 \mathrm{~m}$ e $1.000 \mathrm{~m}$ e profundidade mínima de $11 \mathrm{~m}$.

- Natal - RN: o canal de acesso possui extensão de $2 \mathrm{~km}$, largura entre $90 \mathrm{~m} \mathrm{e}$ $400 \mathrm{~m}$ e profundidade mínima de $5,3 \mathrm{~m}$ e máxima de $7 \mathrm{~m}$.

- Cabedelo - PB: o canal de acesso possui extensão total de $5,5 \mathrm{~km}$, largura mínima de $120 \mathrm{~m}$ e profundidade de $8,5 \mathrm{~m}$.

- Recife - PE: existem 2 canais de acesso ao porto, ambos com características naturais. o principal deles, Canal Sul, possui $3,4 \mathrm{~km}$ de extensão, aproximadamente $260 \mathrm{~m}$ de largura e profundidade de 10,5 m. o outro, denominado Canal Norte, tem cerca de $1000 \mathrm{~m}$ de comprimento, pouca largura e profundidade de $6,5 \mathrm{~m}$ e é utilizado apenas por embarcações de pequeno porte.

- Maceió - AL: o canal de acesso possui $520 \mathrm{~m}$ de comprimento, $80 \mathrm{~m}$ de largura e profundidade de $10 \mathrm{~m}$.

- Barra dos Coqueiros - SE: canal de acesso com comprimento de $550 \mathrm{~m}$, largura de $120 \mathrm{~m}$ e profundidade mínima de $11 \mathrm{~m}$.

- Aratu - BA: o canal de acesso possui extensão de 2 milhas, largura de 180 m e profundidade de $18 \mathrm{~m}$. 
- Salvador - BA: o porto possui 2 canais de acesso. o primeiro chamado canal de dentro, do lado da cidade, com profundidade mínima de 8,00 metros e o segundo chamado de canal de fora, do lado da llha de Itaparica, com profundidade variando entre 13,00 e 55,00 metros.

- Ilhéus - BA: o canal de acesso possui $1000 \mathrm{~m}$ de comprimento, $200 \mathrm{~m}$ de largura com $10 \mathrm{~m}$ de profundidade.

- Barra do Riacho - ES: o canal de acesso tem $670 \mathrm{~m}$ de comprimento, $150 \mathrm{~m}$ de largura e profundidades entre 11,5 e 13,5 m.

- Praia Mole - ES: o canal de acesso tem comprimento de 2 milhas náuticas, $280 \mathrm{~m}$ de largura e 22,5 $\mathrm{m}$ de profundidade.

- Tubarão - ES: o canal acesso tem 2 milhas náuticas de comprimento, $280 \mathrm{~m}$ de largura e $22,5 \mathrm{~m}$ de profundidade.

- Vitória - ES: o canal de acesso se estende por $7 \mathrm{~km}$, com $120 \mathrm{~m}$ de largura e profundidade de $11 \mathrm{~m}$.

- Forno - RJ: o canal de acesso, com extensão de 1,6 km, possui largura mínima de $70 \mathrm{~m}$ e profundidade de $12 \mathrm{~m}$.

- Niterói - RJ: o canal de acesso se estende por $14 \mathrm{~km}$, com largura de $150 \mathrm{~m}$ e profundidade de $12 \mathrm{~m}$.

- Rio de Janeiro - RJ: o canal de acesso compreende $1,5 \mathrm{~km}$ de comprimento, $150 \mathrm{~m}$ de largura mínima e $17 \mathrm{~m}$ de profundidade.

- Sepetiba - RJ: o canal de acesso, com cerca de $22 \mathrm{~km}$, possui largura de $200 \mathrm{~m} \mathrm{e}$ profundidade mínima de $13,5 \mathrm{~m}$.

- Angra dos Reis - RJ: existem dois canais de acesso, um denominado do Sul e outro do Norte, com as seguintes características: Canal do Sul, com comprimento de $8 \mathrm{~km}$, largura de $1,6 \mathrm{~km}$ e profundidade de $14 \mathrm{~m}$; e Canal do Norte, com $11 \mathrm{~km}$ de comprimento, largura de $1,5 \mathrm{~km}$ e a mesma profundidade de $14 \mathrm{~m}$.

- São Sebastião - SP: os canais de acesso correspondentes dispõem, respectivamente, de largura e profundidade de $500 \mathrm{~m}$ e $18 \mathrm{~m}$ (norte) e $300 \mathrm{~m}$ e $25 \mathrm{~m}$ (sul), os dois somando $22,8 \mathrm{~km}$ de extensão.

- Santos - SP: o acesso é franco, contendo um canal com largura de $130 \mathrm{~m}$ e profundidade de $13 \mathrm{~m}$.

- Paranaguá e Antonina - PR: os portos possuem acesso por dois canais, o primeiro - canal Galheta: possui $150 \mathrm{~m}$ de largura e profundidade de $12 \mathrm{~m}$; o segundo é o
Canal Sueste: possui largura de $150 \mathrm{~m} \mathrm{e}$ profundidade de $10 \mathrm{~m}$.

- São Francisco do Sul - SC: o canal de acesso possui extensão de $9,3 \mathrm{~km}$, largura de $150 \mathrm{~m}$ a $175 \mathrm{~m}$ e $10 \mathrm{~m}$ de profundidade.

- Itajaí - SC: o canal de acesso (canal da barra) tem cerca de $1,5 \mathrm{~km}$ de comprimento, largura de $100 \mathrm{~m}$ a $150 \mathrm{~m}$ e profundidade de $8 \mathrm{~m}$.

- Rio Grande - RS: os canais de acesso são o do Porto Novo, que tem comprimento de $5,1 \mathrm{~km}$, largura de $150 \mathrm{~m}$ e profundidade de $8,5 \mathrm{~m}$ e o do Superporto se estende por $4,7 \mathrm{~km}$, com largura mínima de $200 \mathrm{~m}$ e profundidade de $13 \mathrm{~m}$.

\section{ORGANIZAÇÃO E TRATAMENTO DE DADOS}

Com os dados expostos anteriormente, seguiu-se a metodologia descrita abaixo.

Alocados em tabelas, colocou-se os valores de profundidade e largura respectivos de cada Porto. $\mathrm{Na}$ análise do fluxo de cargas considerou-se as cargas mais perigosas.

Pelas informações disponíveis da operação portuária obtém-se as dimensões dos navios.

A metodologia de análise consiste em se traçar dois cenários:

- Um que apresente como resultado final a menor largura requerida pelo navio-tipo, isto é considerando-se as condições mais favoráveis nos diversos quesitos de dimensionamento.

- O outro em contrapartida é o oposto, isto é com a determinação da maior largura requerida, tendo por base as características de dimensionamento desfavoráveis.

As características nos dois casos são explicitadas a seguir com as respectivas justificativas referentes à sua escolha.

\section{Determinação do navio-tipo}

A Tabela 1 apresenta o resultado de naviotipo e carga adotados para cada porto. 


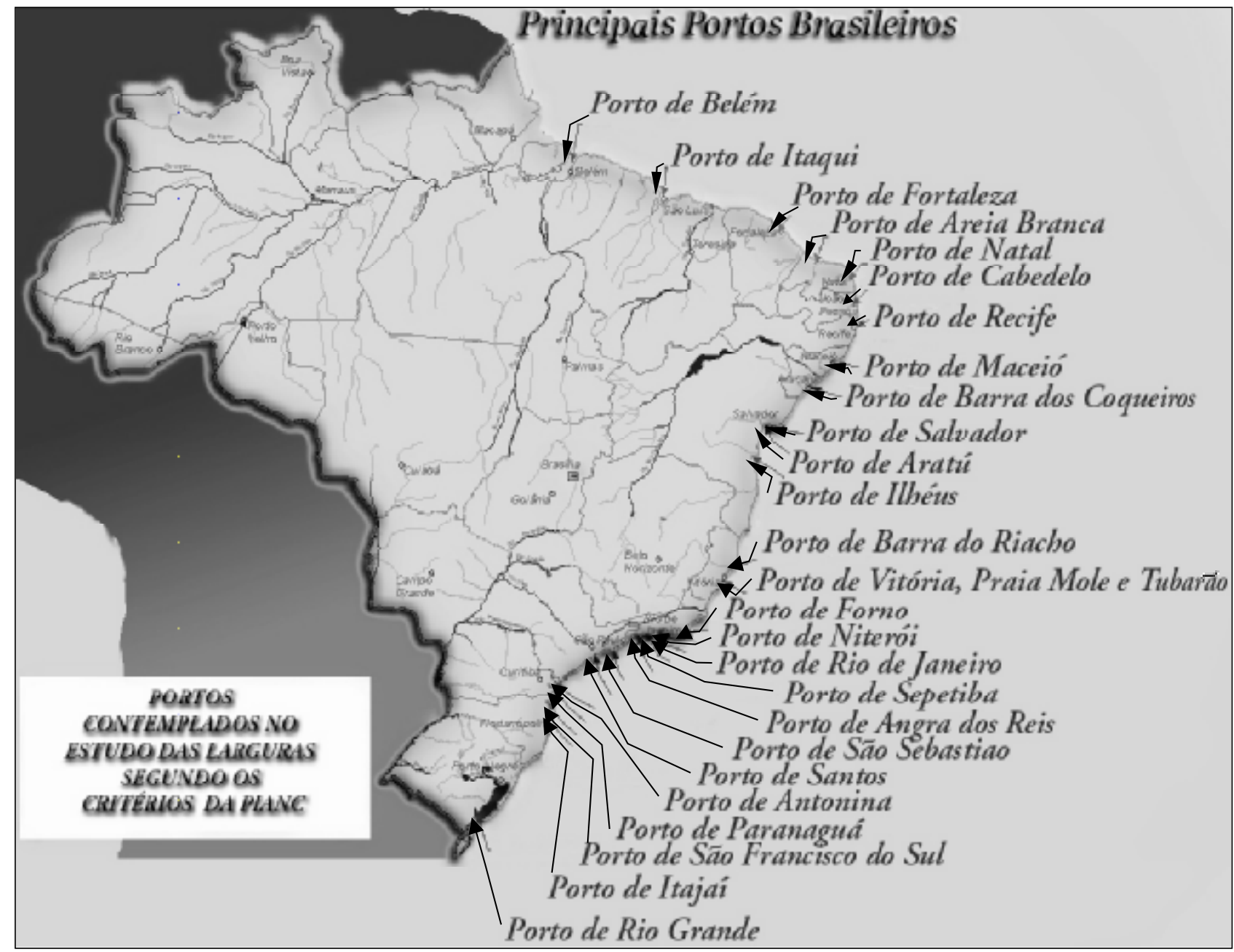

Figura 2. Distribuição geográfica dos portos.

\section{Fatores de incremento de largura em trechos retos com velocidade lenta (entre 5 e 8 nós)}

Manobrabilidade dos navios - A manobrabilidade dos navios é função do coeficiente de bloco da embarcação. Os navios tiveram conservativamente sua manobrabilidade adotada como "ruim" (com coeficiente de bloco muito próximo de um), com incremento de 1,8.B, ou considerados de manobrabilidade "moderada", com incremento de $1,5 . \mathrm{B}$.

\section{Ventos transversais prevalecentes -}

Nos portos da costa brasileira a ocorrência de ventos do tipo severo (acima de 33 nós) são muito baixas, portanto para fins práticos os cenários de melhor e pior caso obedeceram às seguintes classificações:
- Melhor: vento fraco (abaixo de 15 nós) não há incremento de largura;

- Pior: vento moderado - incremento de $0,5 . \mathrm{B}$.

Correntes transversais prevalecentes - Foram consideradas as modalidades Negligenciável (abaixo de 0,2 nós) e Fraca (entre 0,2 e 0,5 nós) para as correntes mais freqüentes. Tem-se:

- Melhor: corrente transversal prevalecente Negligenciável - sem incremento de largura;

- Pior: corrente transversal prevalecente Fraca-0,3.B.

\section{Correntes longitudinais prevalecen-}

tes - Foram consideradas: Fracas (abaixo de 1,5 nó) ou Moderadas (entre 1,5 e 3 nós), portanto: 
- Melhor: corrente longitudinal prevalecente Fraca - sem incremento de largura;

- Pior: corrente longitudinal prevalecente Moderada-0,2.B.

Tabela 1. Navios-Tipo.

\begin{tabular}{|c|c|c|c|c|}
\hline Portos & Carga & $\begin{array}{c}\text { TPB } \\
\left(10^{3} \mathrm{~T}\right)\end{array}$ & $\begin{array}{c}\text { Boca } \\
(\mathrm{m})\end{array}$ & $\begin{array}{c}\mathrm{T} \\
(\mathrm{m}) \\
\end{array}$ \\
\hline Belém & CG & 5 & 15,8 & 6,4 \\
\hline Ponta da Madeira-Itaqui & GS & 380 & 63,5 & 23,0 \\
\hline Mucuripe & GLP & 40 & 30 & 11,8 \\
\hline Areia Branca & GS & 60 & 33,5 & 12,8 \\
\hline Natal & DP & 3 & 13 & 6 \\
\hline Cabedelo & DP & 10 & 19 & 7,8 \\
\hline Recife & GLP & 30 & 28 & 10,8 \\
\hline Maceió & DP & 30 & 28 & 10,8 \\
\hline Barra dos Coqueiros & DP & 30 & 28 & 10,8 \\
\hline Salvador 1 & CG & 15 & 22,6 & 9,2 \\
\hline Salvador 2 & CG & 40 & 30 & 12,5 \\
\hline Aratu & $P Q$ & 50 & 32,2 & 12,6 \\
\hline Ilhéus & DP & 30 & 28 & 10,8 \\
\hline Barra do Riacho & $P Q$ & 50 & 32,2 & 12,6 \\
\hline Praia Mole e Tubarão & $P Q$ & 50 & 32,2 & 12,6 \\
\hline Vitória & CG & 40 & 30 & 12,5 \\
\hline Forno & GS & 40 & 29 & 11,5 \\
\hline Niterói & DP & 3 & 13 & 6 \\
\hline Rio de Janeiro & $P Q$ e $D P$ & 50 & 32,2 & 12,6 \\
\hline Sepetiba & CG & 100 & 39 & 15,3 \\
\hline Angra dos Reis 1 & DP & 80 & 40 & 14 \\
\hline Angra dos Reis 2 & DP & 80 & 40 & 14 \\
\hline São Sebastião 1 & DP & 400 & 68 & 23 \\
\hline São Sebastião 2 & DP & 20 & 24,5 & 9,8 \\
\hline Santos & GL & 50 & 32,2 & 12,6 \\
\hline Paranaguá e Antonina & CG & 50 & 31.7 & 13.5 \\
\hline São Francisco do Sul & CG & 25 & 26,4 & 10,7 \\
\hline Itajaí & $P Q$ & 10 & 19 & 7,8 \\
\hline Rio Grande 1 & $P Q$ & 50 & 32,2 & 12,6 \\
\hline Rio Grande 2 & $P Q$ & 20 & 19 & 7,8 \\
\hline
\end{tabular}

T - Calado; DP - Derivados de Petróleo; PQ - Produtos Químicos; GS - Granéis Sólidos; GL - Granéis Líquidos; CG - Carga Geral; GLP - Gás Liquefeito de Petróleo.

\section{Altura significativa $\left(\mathrm{H}_{\mathrm{s}}\right)$ e comprimen-}

to de onda $(\lambda)$ - Na maior parte do tempo a agitação nos portos da costa brasileira pode ser classificada nos seguintes extremos:

- Melhor: $\mathrm{H}_{\mathrm{s}} \leq 1 \mathrm{~m}$ e $\lambda \leq \mathrm{L}$ (comprimento do navio) - sem incremento de largura;

- Pior: $1 \mathrm{~m}<\mathrm{H}<3 \mathrm{~m}$ e $\lambda>\mathrm{L}-0,5$.B.
Auxílios à navegação - Os auxílios à navegação (sinalização e cartografia) foram considerados: bons e moderados com rara ocorrência de visibilidade pobre, logo:

- Melhor: auxílio à navegação Bom - 0,1.B;

- Pior: auxílio à navegação Moderado 0,2.B.

\section{Características da superfície do fun-}

do do canal - Nos casos estudados a condição de profundidade é menor do que 1,5 vezes o calado em $90 \%$ das situações, o que resume as escoIhas para superfície em:

- lisa e macia;

- lisa ou taludada e rígida;

- rugosa e dura.

Como os canais não apresentam superfície rugosa e dura (por ex.: leito rochoso), as outras duas opções são equivalentes ao incremento proporcionado por elas: 0,1.B. Nas outras situações, de relação profundidade/calado superiores a 1,5 não há incremento.

Profundidade da via navegável - Este item depende da relação entre profundidade e calado. A Figura 3 mostra a distribuição em porcentagem dos navios-tipo, com $87 \%$ de incrementos correspondentes a 0,2 . B, $3 \%$ a 0,1 . B e $10 \%$ sem incremento de largura.

Periculosidade da carga - O risco da carga foi caracterizado em baixo, médio e alto, correspondendo, respectivamente, a incrementos de 0 , 0,5.B e 1,0.B.

Intensidade de tráfego - A intensidade de tráfego, aplicada apenas em canais de mão dupla, foi considerada:

- Melhor: leve (até 1 navio por hora) - sem incremento de largura;

- Pior: Moderada (de 1 a 3 navios por hora) incremento de 0,2.B.

\section{Largura adicional devido à folga com} a margem - As laterais de todos os canais de acesso foram consideradas como: taludadas e com bancos de areia, o que corresponde a um incremento de 0,3.B. 


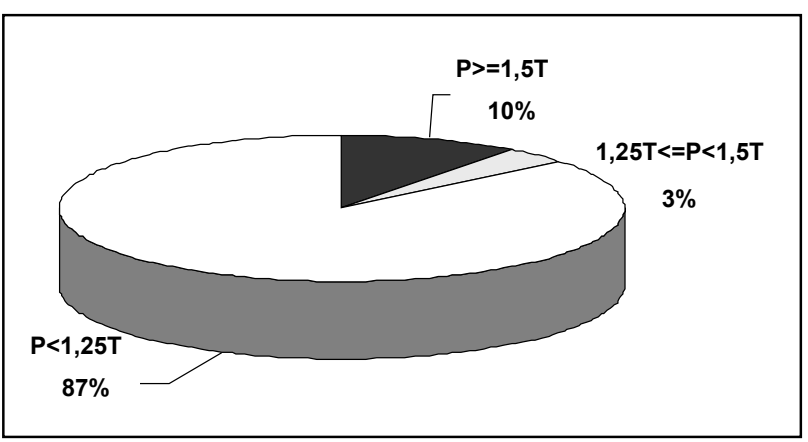

Figura 3. Distribuição dos navios-tipo quanto à relação profundidade (P)/calado (T)

Largura de passagem para canais de mão dupla - O critério para classificação dos canais é dado pela Tabela 2:

Tabela 2. Critério de classificação de canais de acesso (PIANC, 1997).

\begin{tabular}{cc}
\hline Se largura do canal & Canal de \\
\hline$<6 . B$ & mão simples \\
$>6 . B$ & mão dupla \\
\hline
\end{tabular}

Para este incremento obteve-se 1,2.B.

\section{Resumo de fatores de incremento de largura}

A Tabela 3 apresenta o resumo dos fatores de incremento de largura obtidos.

Os canais foram avaliados como de mão simples ou dupla de acordo com o critério sugerido pela PIANC (1997).

\section{APRESENTAÇÃO DE RESULTADOS}

$\mathrm{Na}$ Tabela 4 e na Figura 4 estão apresentados os resultados referentes aos cálculos com os índices relacionados na Tabela 3.

\section{CONCLUSÕES}

Da Tabela 4 e Figura 4 têm-se que:

- $22 \%$ dos portos não atenderam aos requisitos necessários determinados pela PIANC para o cenário de pior caso, conforme apresentado na Figura 5. No cenário de pior
Tabela 3. Resumo de coeficientes de incremento de largura.

\begin{tabular}{|c|c|c|}
\hline \multirow[t]{2}{*}{ Fator } & \multicolumn{2}{|c|}{ Coeficientes } \\
\hline & Melhor & Pior \\
\hline Manobrabilidade; & $1,5 \mathrm{~B}$ & $1,8 \mathrm{~B}$ \\
\hline Velocidade do Navio; & 0 & 0 \\
\hline $\begin{array}{l}\text { Ventos Transversais } \\
\text { Prevalecentes; }\end{array}$ & 0 & $0,5 B$ \\
\hline $\begin{array}{l}\text { Correntes Transversais } \\
\text { Prevalecentes; }\end{array}$ & 0 & $0,3 \mathrm{~B}$ \\
\hline $\begin{array}{l}\text { Correntes Longitudinais } \\
\text { Prevalecentes; }\end{array}$ & 0 & $0,2 B$ \\
\hline $\begin{array}{l}\text { Altura Significativa Hs e } \\
\text { Comprimento de Onda }(\lambda)\end{array}$ & 0 & $0,5 B$ \\
\hline Auxílios à Navegação; & $0,1 \mathrm{~B}$ & $0,2 \mathrm{~B}$ \\
\hline Superfície de Fundo; & 0 & $0,1 \mathrm{~B}$ \\
\hline Profundidade da Via Navegável; & \multicolumn{2}{|c|}{ Variável } \\
\hline Nível de Periculosidade da Carga; & \multicolumn{2}{|c|}{ Variável } \\
\hline Intensidade de Tráfego; & 0 & $0,2 \mathrm{~B}$ \\
\hline $\begin{array}{l}\text { Largura devido à folga com a } \\
\text { margem; }\end{array}$ & $0,3 \mathrm{~B}$ & $0,3 \mathrm{~B}$ \\
\hline $\begin{array}{l}\text { Largura de Passagem para canais } \\
\text { de mão dupla; }\end{array}$ & $1,2 \mathrm{~B}$ & $1,2 \mathrm{~B}$ \\
\hline
\end{tabular}

caso destes canais apenas um não apresenta carga de alta periculosidade;

- $100 \%$ dos portos atendeu aos requisitos que tratavam de análise de melhor caso.

- Os canais de acesso dos portos brasileiros apresentam na maior parte dos casos uma largura bastante elevada, o que conduz sempre à aceitação pelos critérios estabelecidos pela PIANC.

- Os portos que apresentaram rejeição quanto ao critério têm como conseqüência uma sub-utilização de sua profundidade, provocando assim restrições quanto aos tamanhos dos navios e onerando o processo de transporte.

- Outro fator importante é a análise da ocorrência das larguras mínimas, isto é, saber em qual porcentagem do comprimento ela ocorre. Se esta porcentagem for pequena, num canal de mão dupla, uma solução seria fazer desse trecho um canal de mão única e aumentar um pouco a velocidade das embarcações no trecho, compensando a deficiência operacional produzida pela singularidade. 
Tabela 4. Análise comparativa de largura.

\begin{tabular}{|c|c|c|c|c|c|c|c|}
\hline PORTOS & $\begin{array}{c}\text { Boca } \\
(\mathrm{m})\end{array}$ & $\begin{array}{l}\text { Largura do } \\
\text { canal real } \\
\quad(\mathrm{m})\end{array}$ & $\begin{array}{l}\text { Largura do } \\
\text { canal real } \\
W=\alpha_{\text {real }}{ }^{*} B\end{array}$ & $\begin{array}{c}\text { Largura mínima } \\
\text { PIANC } \\
\text { Wmin }=\alpha_{\text {min }}{ }^{*} B\end{array}$ & $\begin{array}{l}\text { Largura máxima } \\
\text { PIANC } \\
\text { Wmáx }=\alpha_{\text {máx }}{ }^{*} B\end{array}$ & $\begin{array}{c}\text { Razão } \\
\mathrm{W}_{\text {máx }} / \mathrm{W}\end{array}$ & $\begin{array}{l}\text { Razão } \\
\text { W }_{\text {min }} / \mathrm{W}\end{array}$ \\
\hline & B & W & $\alpha_{\text {real }}$ & $\alpha_{\min }$ & $\alpha_{\text {máx }}$ & $W_{\text {razão.máx }}$ & $W_{\text {razão.min }}$ \\
\hline Belém & 15,8 & 90,00 & 5,70 & 1,70 & 3,38 & 0,59 & 0,30 \\
\hline P. Madeira/Itaqui & 63,5 & 500,00 & 7,90 & 2,30 & 5,40 & 0,70 & 0,30 \\
\hline Mucuripe & 30 & 100,00 & 3,33 & 2,70 & 4,34 & 1,30 & 0,81 \\
\hline Areia Branca & 33,5 & 400,00 & 11,94 & 4,10 & 5,73 & 0,48 & 0,34 \\
\hline Natal & 13 & 90,00 & 6,92 & 5,10 & 6,79 & 0,98 & 0,74 \\
\hline Cabedelo & 19 & 120,00 & 6,32 & 5,10 & 6,77 & 1,07 & 0,81 \\
\hline Recife & 28 & 260,00 & 9,29 & 5,10 & 6,74 & 0,73 & 0,55 \\
\hline Maceió & 28 & 80,00 & 2,86 & 2,70 & 4,34 & 1,52 & 0,95 \\
\hline Barra dos Coqueiros & 28 & 120,00 & 4,29 & 2,20 & 3,84 & 0,90 & 0,51 \\
\hline Aratu & 32,2 & 180,00 & 5,59 & 2,60 & 3,65 & 0,65 & 0,47 \\
\hline Salvador 1 & 22,6 & 200,00 & 8,85 & 4,10 & 5,24 & 0,59 & 0,46 \\
\hline Salvador 2 & 30 & 200,00 & 6,67 & 4,10 & 5,24 & 0,79 & 0,62 \\
\hline llhéus & 30 & 200,00 & 6,67 & 5,10 & 6,73 & 1,01 & 0,77 \\
\hline Barra do Riacho & 32,2 & 150,00 & 4,66 & 2,90 & 4,53 & 0,97 & 0,62 \\
\hline Praia Mole e Tubarão & 32,2 & 280,00 & 8,70 & 4,90 & 6,56 & 0,75 & 0,56 \\
\hline Vitória & 30 & 120,00 & 4,00 & 1,70 & 3,33 & 0,83 & 0,43 \\
\hline Forno & 29 & 70,00 & 2,41 & 1,70 & 3,34 & 1,38 & 0,70 \\
\hline Niterói & 13 & 70,00 & 5,38 & 2,70 & 4,39 & 0,81 & 0,50 \\
\hline Rio de Janeiro & 32,2 & 150,00 & 4,66 & 2,60 & 4,24 & 0,91 & 0,56 \\
\hline Sepetiba & 39 & 200,00 & 5,13 & 1,70 & 3,33 & 0,65 & 0,33 \\
\hline Angra dos Reis 1 & 40 & 1600,00 & 40,00 & 5,10 & 6,73 & 0,17 & 0,13 \\
\hline Angra dos Reis 2 & 40 & 1500,00 & 37,50 & 5,10 & 6,73 & 0,18 & 0,14 \\
\hline São Sebastião 1 & 68 & 300,00 & 4,41 & 2,90 & 4,52 & 1,02 & 0,66 \\
\hline São Sebastião 2 & 24,5 & 550,00 & 22,45 & 5,10 & 6,74 & 0,30 & 0,23 \\
\hline Santos & 33,5 & 210,00 & 6,30 & 4,50 & 6,90 & 1,10 & 0,70 \\
\hline Antonina e Paranaguá & 31,7 & 150,00 & 4,70 & 1,20 & 3,10 & 0,70 & 0,30 \\
\hline São Francisco do Sul & 26,4 & 150,00 & 5,68 & 1,70 & 3,34 & 0,59 & 0,30 \\
\hline Itajaí & 19 & 100,00 & 5,26 & 2,70 & 4,36 & 0,83 & 0,51 \\
\hline Rio Grande 1 & 32,2 & 200,00 & 6,21 & 5,10 & 6,73 & 1,08 & 0,82 \\
\hline Rio Grande 2 & 19 & 150,00 & 7,89 & 5,10 & 6,76 & 0,86 & 0,65 \\
\hline
\end{tabular}




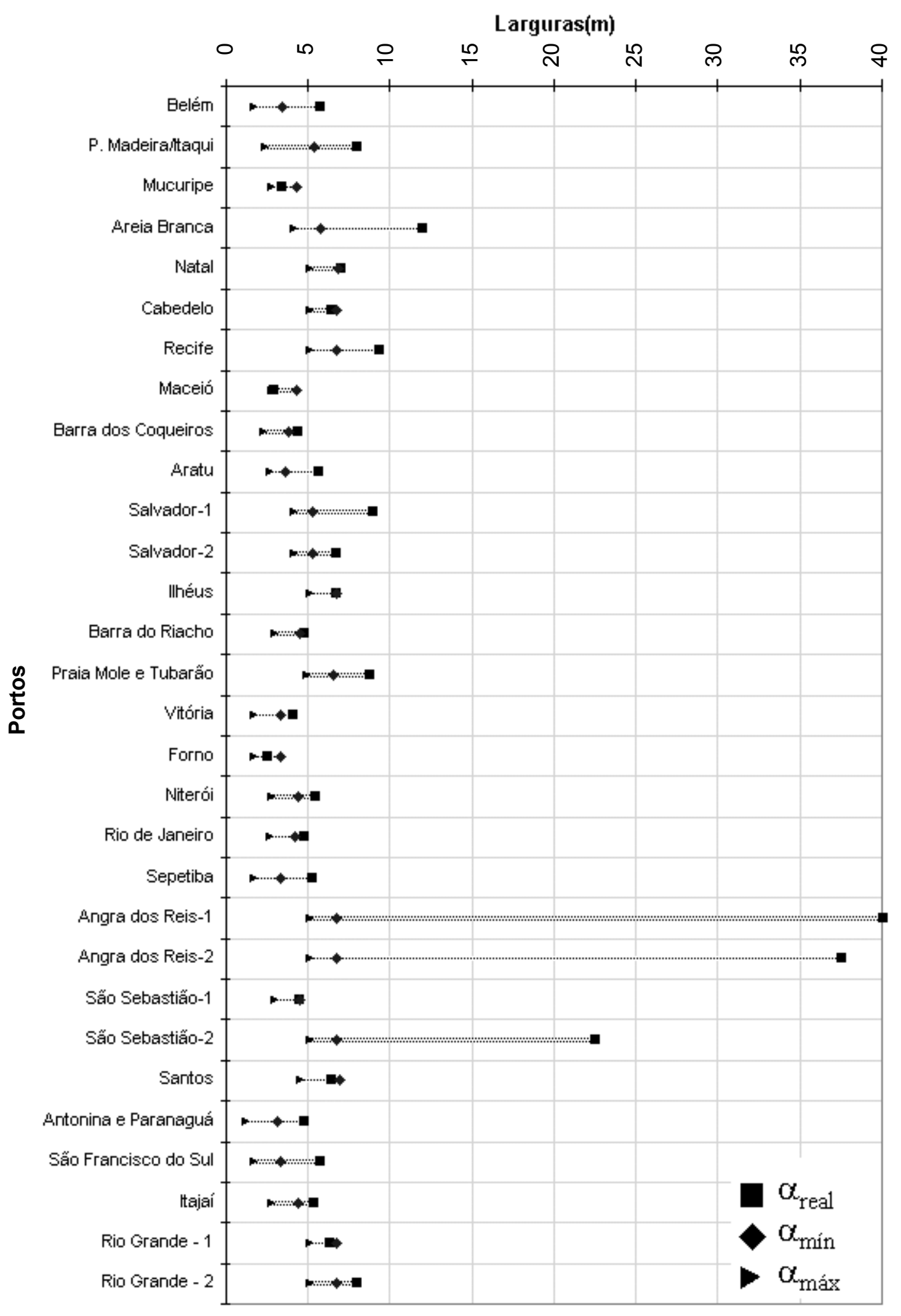

Figura 4. Análise comparativa de larguras. 
Porcentagem de Aprovação no Critério PIANC, diante do Cenário de Pior Caso

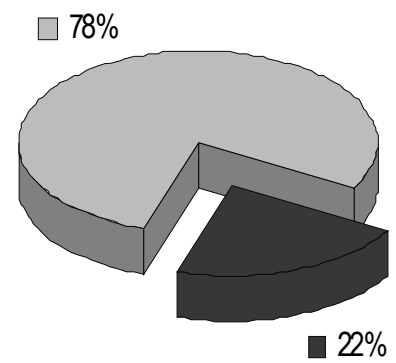

$\square$ Não Aprovados $\square$ Aprovados

Figura 5. Porcentagem de aprovação com cenário de pior caso

\section{REFERÊNCIAS}

BRASIL/MINISTÉRIO DOS TRANSPORTES (1999) Sites da Internet: Ministério dos Transportes: www.transportes.gov.br; Companhia de Docas da Bahia (CODEBA): www.codeba.com.br; Companhia de Docas do Pará (CDP): www.cdp.com.br; Companhia de Docas do Espírito Santo (CODESA): www.codesa.com.br

PIANC (1997) "Approach Channels - A guide for design". Final Report of the Joint Working Group II-30, Supplement to Bulletin 95, Brussels and Tokyo.

\section{Outer Approach Channel of Brazilian Ports According to PIANC Guidelines- Width Evaluation}

\section{ABSTRACT}

Project design standardization of port approach channels for a design ship or ships presents guidelines for channel design features. This design is based on the evaluation of an existing or proposed channel according to the design premises or their modifications, considering safety criteria. The concept design basically consists of the definition of width, alignment and depth dimensions. Developments in commercial and leisure navigation are continually stimulating appropriate designs for approach channels to avoid delays, congestions, incidents and accidents which result in heavy penalties for the port's economy. The project design study, based on initial physical environmental data, a design ship and other considerations and forecasts, is the first stage of the approach channel width rational determination. This paper presents the results of the evaluation of thirty outer approach channels of the main Brazilian ports according to PIANC guidelines. 